\title{
Design of a small logistics robot system based on solar energy
}

\author{
Hong Chen \\ School of Information Engineering, Wuhan University of Technology, Wuhan, China
}

\begin{abstract}
In recent years, with the rise of various take-out and express companies, small-scale logistics such as take-out has become an important part of people's daily life, and riders shuttle between major campuses and communities, generating energy from the distribution of battery cars and motorcycles. Consumption, exhaust pollution, and campus traffic accidents. In response to this situation, this paper designs and implements a small solar-based logistics robot, hoping to make campus takeaway delivery more energy-efficient, environmentally friendly, safer, smarter and more efficient.
\end{abstract}

Keywords: solar energy, logistics, system design

This article aims to design a low-power small logistics robot to replace the rider in the community and campus for the problem of energy consumption and environmental pollution during the transportation of battery cars and motorcycles. This robot is powered by a highly efficient light-following solar base station, using sensors such as cameras and lidars and improved processing algorithms to make robot delivery safer, and it can carry multiple items at one time while working, and deliver them sequentially according to the optimal path, which is more intelligent Efficient.

\section{Design background}

In recent years, the food delivery industry has risen rapidly. There are a large number of food delivery riders traveling around the campus every day. While bringing convenience to the teachers and students in the school, it also creates a series of energy and safety issues that need to be solved urgently.

According to the "Monitoring Report on China's Online Food Delivery Market in 2018", in 2018, the number of daily food orders for Chinese universities exceeded 9 million, and the delivery vehicles were mainly battery cars and motorcycles, with a ratio of about 10:1. According to the average driving distance of 1.4 kilometers on campus, colleges and universities across the country travel more than 12.6 million kilometers every day. Therefore, campus takeaway delivery causes a large amount of electricity and gasoline consumption, as well as air pollution that cannot be ignored.

In terms of safety, some takeaway riders blindly pursue delivery speed and make calls on bicycles, resulting in frequent traffic accidents on campus. According to the data released by the Wuhan traffic police, there were 97 campus traffic accidents caused by 
takeaway delivery in Wuhan in 2017, and there are currently more in the country. Colleges and universities have restricted takeaway vehicles from entering campuses.

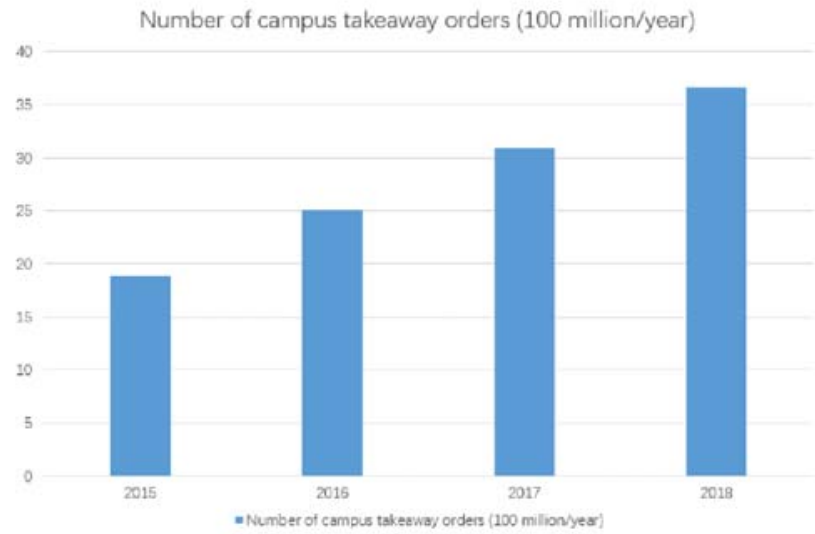

Figure 1 Number of campus takeaway orders(100 million/year)

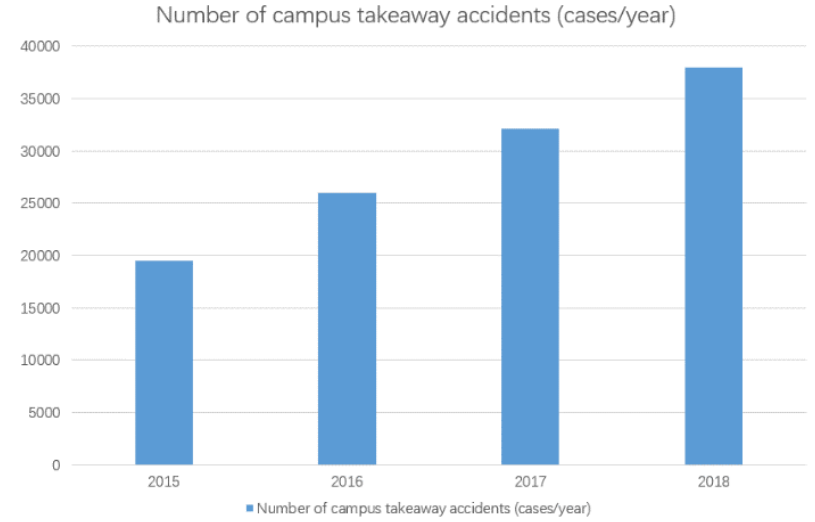

Figure 2 Number of campus takeaway accidents

\section{Technical route}

Aiming at the current pollution, safety and energy consumption problems of food delivery, this paper proposes an unmanned food delivery robot based on light-following solar energy and path planning. The specific technical route is as follows:

(1) Research the lidar avoidance algorithm and the binocular camera image processing algorithm to provide a strong guarantee for solving the safety problem of delivery of food on campus.

(2) Study the absolute position reference correction method to improve GPS accuracy and enhance its reliability.

(3) Research and refer to the various optimal path algorithms to provide an algorithm basis for the delivery path of the takeaway robot, improve the operating efficiency of the robot, shorten the delivery time, and save energy.

\section{Technical realization plan}


Aiming at the problems of energy consumption and frequent safety accidents caused by traditional food delivery, this paper, after in-depth research, proposes an improvement based on the existing unmanned vehicle scheme, and designs a food delivery robot to make it more suitable for campus environments. Figure 3 shows the overall structure of the robot.

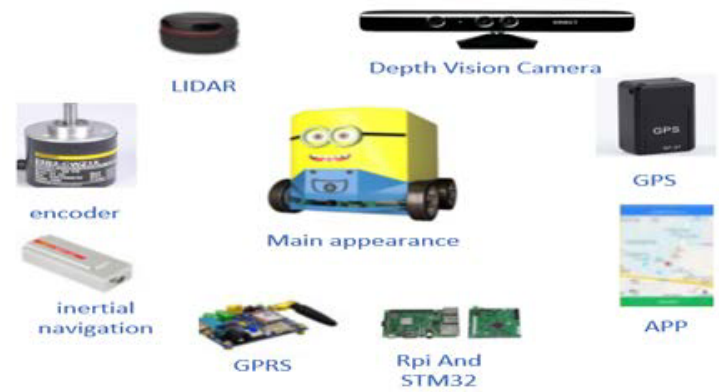

Figure 3 The overall structure of the robot

\subsection{Improved Lidar Obstacle Avoidance Algorithm}

Judging the dynamic and static obstacles in front of the robot is a prerequisite for the safe delivery of the robot. In this paper, a low-cost single-line lidar is used to perceive obstacle information, build an obstacle avoidance model, and refer to the existing mainstream obstacle avoidance algorithms to propose an obstacle avoidance control algorithm combining fuzzy control and potential field. The algorithm overcomes the security problems caused by the shock caused by the traditional virtual potential field to a certain extent. The robot divides the front into braking zone, obstacle avoidance zone, deceleration zone and rectangular detection zone. When point cloud data is detected in the monitoring area, the robot enters deceleration and obstacle avoidance mode. When the robot enters the obstacle avoidance mode, the robot's braking zone will be converted into a compressed braking zone until it exits the obstacle avoidance mode.

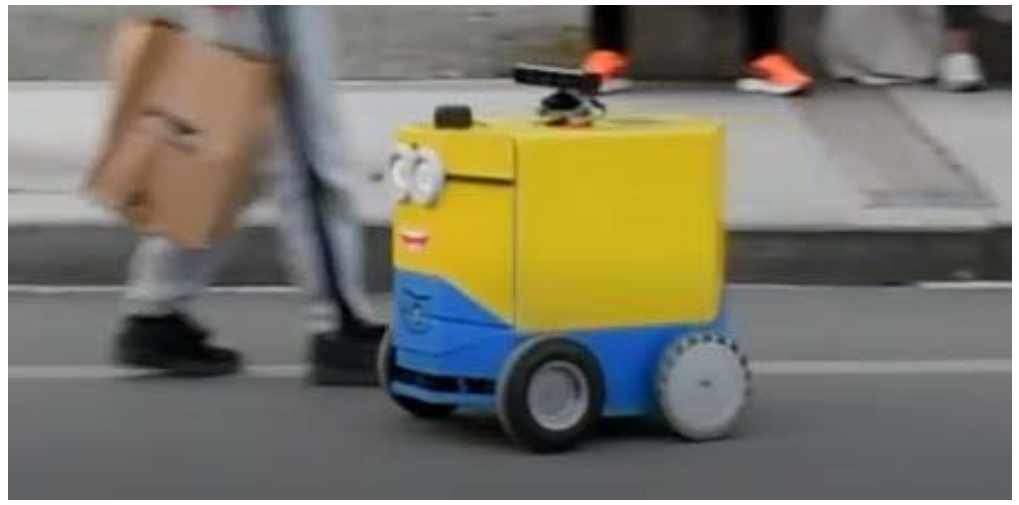

Figure 4 Obstacle avoidance test of experimental machine

\subsection{Binocular camera recognition solution}

In order to effectively identify road obstacles and ensure delivery safety, this paper uses binocular cameras to collect road information, at the same time builds a hardware platform based on ROS + embedded, performs image processing based on OPENCV, uses Hough transform and Canny operator for road recognition, and uses HoG feature+ The SVM 
classifier performs human body recognition. When the robot detects the presence of a pedestrian in front, it will automatically avoid and buzzer to warn. Figure 4 is an example of the obstacle avoidance test of the experimental machine.

\subsection{Absolute position reference correction method}

In this paper, based on Kalman filter sensor fusion technology, GPS and inertial sensor data are fused to achieve a relatively real-time positioning effect. In order to solve the problem of poor GPS positioning, this article uses the absolute position reference correction method, through which the GPS positioning error $\triangle$ at a certain time is calculated, and the GPS coordinates at that time are compensated to obtain more accurate GPS coordinate data. Figure 5 is a flow chart of the implementation of calculating accurate GPS coordinates using the absolute position reference correction method.

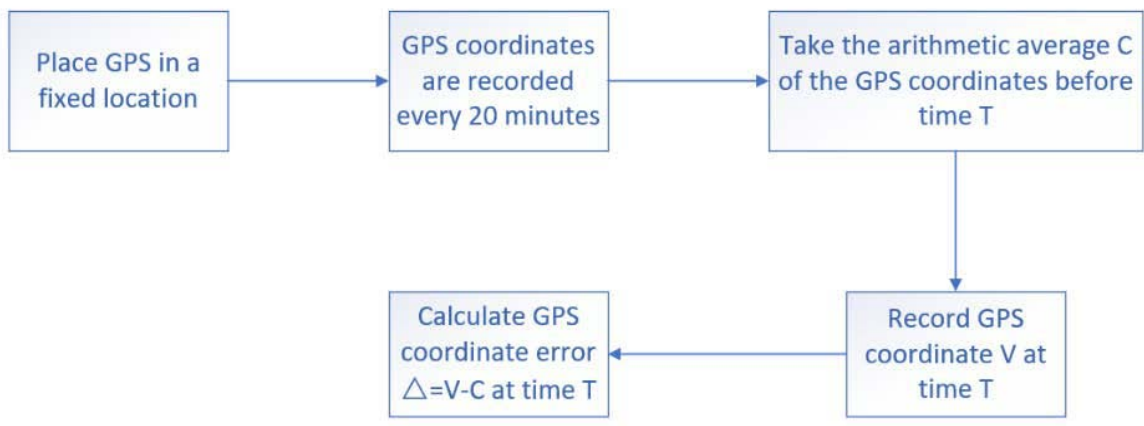

Figure 5 Flow chart of calculating precise coordinates

\subsection{Best path algorithm}

The best path algorithm used in this article is ant colony algorithm. The research model of ant colony algorithm comes from the simulation of ants' foraging behavior. Suppose $\mathrm{m}$ ants are placed in $\mathrm{n}$ random delivery points, $\mathrm{C}$ is the set of locations, $\tau_{-} \mathrm{ij}(\mathrm{t})$ represents the amount of information on the path of points $\mathrm{i}$ and $\mathrm{j}$ at time $\mathrm{t}$, and $\eta_{-} \mathrm{ij}$ is a heuristic factor, which means that the ants from point $i$ at time $t$ The expected distance to move to point $j$, usually $\eta_{-} \mathrm{ij}$ is the reciprocal of the distance between points. Each ant independently selects the next location to be transferred according to the pheromone amount of each path, and records the community that ant $\mathrm{k}$ has walked in the tabu_k table. The state transition probability $p_{-} i j^{\wedge} k(t)$ of ant $k$ moving from point $i$ to point $j$ at time $t$ is shown in formula (1).

$$
p_{i j}^{k}(t)=\left\{\begin{array}{c}
\text { allowed }_{k}=\left\{C-\text { tabu }_{k}\right\} \\
\frac{\left[\tau_{i j}(t)\right]^{a} g\left[\eta_{i j}(t)\right]^{\beta}}{\sum_{s \in \text { allowed, },\left[\tau_{i s}(t)\right]^{a} g\left[\eta_{i s}(t)\right]^{\beta}}}, \text { if } j \in \text { allowed }_{k} \\
0
\end{array}\right.
$$

In the formula, a is the information heuristic factor, which reflects the influence of pheromone on the path chosen by the ant, $\beta$ is the expected heuristic factor, which indicates the influence of the path length on the ant, and "allowed"_k is the choice when ant k moves next Collection of locations.

Pheromone update. After all ants have completed a traversal, the remaining pheromone 
on the path is updated, and the pheromone on each path is adjusted according to formula (2).

$$
\left\{\begin{array}{c}
\tau_{i j}(t+n)=(1-\rho) \cdot \tau_{i j}(t)+\Delta \tau_{i j}(t) \\
\Delta \tau_{i j}(t)=\sum_{k=1}^{m} \Delta \tau_{i j}^{k}(t)
\end{array}\right.
$$

In the formula, $\rho$ is the volatilization coefficient, which reflects the persistence of the pheromone. The value of $\rho$ is between 0 and 1. $\Delta \tau$ ij (t) represents the increase of pheromone on the path $(\mathrm{i}, \mathrm{j})$ after this cycle. $\Delta \tau_{-} \mathrm{ij}^{\wedge} \mathrm{k}(\mathrm{t})$ represents the amount of pheromone left by the kth ant on the path $(i, j)$.

$$
\Delta \tau_{i j}^{k}(t)=\left\{\begin{array}{c}
\frac{Q}{L_{k}}, \text { if the kth ant passes }(i, j) \text { in this cycle } \\
0, \text { others }
\end{array}\right.
$$

In the formula, $\mathrm{L}_{-} \mathrm{k}$ is the sum of the distance traveled by the kth ant in this cycle, and Q is the total amount of pheromone released by the ant after completing a path search. The model uses the entire information after the ants completes a cycle to update the pheromone on the path.

\section{Conclusion and prospect analysis}

With the development of artificial intelligence and the advent of the $5 \mathrm{G}$ era, the problem of $\mathrm{R} \& \mathrm{D}$ and production of robots and related products on software and hardware platforms has been solved. In "Made in China 2025", the country regards robots as a key development object, and has policy support for the design and development of take-out robots and industrial supporting facilities. Moreover, the food delivery market now has a huge volume and continuous increase, but the current food delivery related industries have traffic safety, energy costs and other issues. Therefore, there is a huge demand for small logistics robot products in the market, and related R\&D and design can also be marketed and policy-driven. stand by. The small solar-based logistics robot proposed in this article solves the safety problems on campus in the takeaway industry and the energy problems in the takeaway delivery process. The robot design and development process and related technology use in this article also have certain implications for robot development in other fields. Enlightening.

\section{References}

1. Li Peiying, Liu Weiliang. Research on the design of mobile robot control system based on automatic guidance[J]. Science and Technology Innovation, 2020, (29): 63-66.

2. Zhang Li, Fan Xinyue. Patrol key route planning based on ant colony algorithm[J]. Mathematics in Practice and Knowledge, 2020,50(16):144-152.

3. Lu Jifeng, Luo Lei, Shilun. Error calibration method of differential mobile robot sensor system based on UMBmark and EKF[J]. Mechanical Design and Research, 2020, 36(04): 149-153+165.

4. Chen Weili, Jia Chao. The edge detection of laser imaging radar image based on artificial intelligence technology [J]. Laser Magazine, 2020, 41(06): 85-88.

5. Liu Yiqing. The development and application of takeaway robot technology[J]. Science and Technology Economy Market, 2020, (06): 14-16. 